\title{
1 Effects of verbal instruction vs modelling on (over)imitation and cumulative culture
}

7 Abstract

8

\author{
Aliki Papa, Psychology, Heriot-Watt University. A.Papa@hw.ac.uk \\ Mioara Cristea, Psychology, Heriot-Watt University. M.Cristea@hw.ac.uk \\ Nicola McGuigan, University of Western Scotland. Nicola.McGuigan@uws.ac.uk \\ Mónica Tamariz, Psychology, Heriot-Watt University. M.Tamariz@hw.ac.uk
}

Human culture is the result of a unique cumulative evolutionary process. The social transmission mechanisms underlying this process are still not fully understood. In particular, the role of language - another unique human behaviour - in social transmission is underexplored. In this first direct, systematic comparison of demonstration vs language-based social learning, we measured the transmission fidelity and cumulative improvement of an action sequence whose objective was to extract a reward from a box. Participants were organised in transmission chains, and each of them either watched a model demonstrate an action sequence or listened to verbal instructions to produce the action sequence. In order to explore imitation and overimitation, the sequences included actions that were causally relevant or irrelevant, respectively, to extracting the reward. We explored these effects in transmission adults and in 6 to 8-year-old children. Overall, we found more retention of causally relevant than irrelevant actions. More interestingly, children (but not adults) retained more causally irrelevant actions under verbal instruction, but more causally relevant actions under demonstration. Cumulative cultural evolution produces sophisticated, complex behaviour whose function may not be obvious. By promoting the retention of behaviour even when its function is not understood, specifically in children, language may play a supportive role in cumulative cultural evolution.

\section{Introduction}

Human culture - our complex, refined behaviours, technologies, institutions, ideas etc - is the unique product of cumulative cultural evolution (Boyd and Richerson 1996). Tomasello (1999) introduced the term 'ratchet effect': modifications to a cultural trait can be retained and 
beneficial changes can accumulate to yield products of such sophistication as no single individual or generation could accomplish on their own (Tomasello et al. 1993). Legare and colleagues emphasise the 'dual engines' of cumulative transmission, namely imitation and innovation. The former allows the transmission of a vast array of cultural traits from generation to generation and eliminates the costs of individual learning by trial and error, and it is the focus of this paper; the later enables cultural adaptation to new environments (Legare and Nielsen 2015; Legare and Harris 2016). In order to support cumulative cultural evolution, transmission of information between individuals must have a high degree of fidelity (Tomasello 1994, 1999; Tennie et al. 2009). Imitation, a type of social learning that focuses on faithfully copying the actions observed rather than their outcomes, is crucial for faithful transmission (Tomasello 1990; Whiten and Ham 1992; Meltzoff 2007; Tennie et al. 2009) as it allows learners to acquire skills even if they do not fully understanding the goal of each action (Horner and Whiten 2005; Gergely and Csibra 2006; Tamariz 2019).

Language, a uniquely human behaviour that mediates communication and supports cooperation and collaboration (Tomasello 2006) and teaching (Laland 2017), has the potential to boost the transmission fidelity of knowledge, attitudes, beliefs and values beyond what can be learned through the observation of actions and outcomes. In spite of this, the vast majority of social transmission studies involve observational learning, in which a demonstrator performs an action for an observer, who then goes on to attempt it. This approach suits developmental as well as cross-species comparative goals, but neglects the role of language as a potential mainstay of human cumulative cultural evolution.

Several recent studies of social learning have incorporated verbal instruction to their designs in addition to demonstration. In one condition of Dean et al.'s (2012) study, 3 and 4 year-old children learned how to extract a reward from a puzzle box by observing other children. Some of the demonstrators spontaneously provided instructions, and learners who received these instructions significantly outperformed those who did not. Dean et al. (2012) therefore linked language (among other factors) and cumulative improvement. Zwirner and Thornton's (2015) participants had to construct baskets out of everyday materials that could carry as much rice as possible. The authors found that teaching was not essential for cumulative improvement, although its presence resulted in more robust baskets than when they only observed others' actions (imitation) or only observed the finished product (emulation). Caldwell and Millen 
(2009) evaluated the contribution of different types of social learning to cumulative improvement using a task in which participants had to make paper aeroplanes that would fly as far as possible. They systematically manipulated whether participants could do imitation, emulation and receive teaching, which included a verbal component, and found that performance improved in all conditions to similar extents. Prior familiarity with the task, however, may have confounded the results. Action familiarity may bias cultural transmission, but it is not clear how. Meltzoff $(1988 a, 1988 b)$ found that 14-month-olds tended to reproduce novel actions, but in Reader et al.'s studies (2007), participants tended to follow a familiar route over a novel one, even if it was costlier.

A group of studies have examined the effects of verbal instruction on social transmission using stone-tool manufacturing tasks. Putt et al. (2014) had participants learn how to make bifaces and found that adding verbal instructions to demonstration of an action sequence increased copying fidelity, as it increased the probability that novice participants would reproduce the instructor's exact actions. However, language made no significant difference in terms of skill, i.e. how well participants learned the target behaviour. In the demonstration-only group, individual actions were more efficient. The conclusion that language does not necessarily enhance transmission is in line with a similar study by Ohnuma et al. (1997). Here, participants' production of Levallois stone tools did not differ between a demonstration-plusverbal-instruction and a demonstration-only group. In contrast with those studies, in Morgan et al.'s (2015) comparison of five transmission mode conditions (involving demonstration, gestural communication and verbal communication) in a flint flake manufacture task, participants in the 'verbal teaching' condition performed significantly better than in most other conditions, and slightly better than participants in the 'gestural teaching' condition.

In these studies verbal transmission was never tested in isolation. The verbal or teaching conditions often involved face to face interaction, during which participants engaged in both verbal and gestural interaction (Dean et al. 2012; Caldwell and Millen 2009; Zwirner and Thornton 2015). Morgan et al. (2015) included distinct gestural and verbal teaching conditions; however, in both cases verbal input was provided in addition to observation. The effects of verbal instruction and observational learning on social transmission have, to our knowledge, never been directly compared. 
The skills transmitted in social learning studies typically include causally relevant actions, which are necessary to the completion of the task, and causally irrelevant actions, which are not required for task completion. Comparative studies show that humans do "blanket copying", i.e. they copy the actions they see no matter whether those are causally relevant ('imitation') or irrelevant ('overimitation', Nielsen 2006; Lyons et al. 2007, see Hoehl et al. 2019 for recent review) and irrespective of whether they have information about their causality or not (Whiten et al. 2009). Other primates, in contrast, tend to omit actions known to be causally irrelevant from their output (Horner and Whiten 2005). Cross-cultural studies reveal that overimitation is present across a variety of world cultures (Nielsen and Tomaselli 2010; Nielsen et al. 2014; for an exception in the Aka children, see also Berl and Hewlett 2015; Clegg and Legare 2016). As an enabler of faithful action transmission unbiased by causal relevance or efficiency, overimitation plays a key role in cumulative cultural evolution (Legare and Nielsen 2015; Tomasello 2016; Nielsen 2018; Tamariz 2019).

Overimitation does not always have the same degree of fidelity. Rather, children are capable of doing flexible imitation (Clegg and Legare 2017) according to the nature of the task. Causally opaque actions presented as conventional, or 'social 'rituals' (Hermann et al. 2013; Legare et al. 2015; Clegg and Legare 2015) and actions that are 'playful' (Nielsen et al.2012) are copied with higher fidelity than instrumental actions that achieve a functional goal. This difference seems to decrease with age, as older children copy conventional and instrumental causally irrelevant actions with similar fidelity (Moraru et al. 2016).

Children are high fidelity imitators and overimitators (Nagell et al. 1993; Horner and Whiten 2005; Call et al. 2004; Tennie et al. 2006; McGuigan et al. 2007; McGuigan and Whiten 2009; McGuigan et al. 2011; Flynn 2008; Flynn et al. 2012; Haun et al. 2014), as are adults (McGuigan 2012, Whiten et al. 2016). The tendency to overimitate seems to increase with age, as older children copy causally irrelevant actions more than younger children (McGuigan et al. 2007) and adults more than children (McGuigan et al. 2011; McGuigan 2012).

A common experimental paradigm used to explore how social learning supports cultural accumulation involves transmission chains in which the actions reproduced by a participant are shown to the next participant in the chain. This paradigm amplifies the effects of transmission biases (Kirby et al. 2008). Studies with children show that, along the chain, 
innovations are introduced and causally irrelevant actions are omitted to a higher extent than causally relevant ones, which results in a cumulative increase in efficiency or adaptiveness in most cases (Flynn 2008; McGuigan and Graham 2009; McGuigan 2012; Tennie et al. 2014; McGuigan et al. 2017).

We designed a study directly to compare for the first time the role of two transmission modes - verbal instruction and demonstration - on social transmission of relevant and irrelevant actions and on cumulative improvement of efficiency in children and adults. We used an existing experimental task and apparatus in which participants had to extract a reward from a box, and arranged our participants in transmission chains. We will explore all the interactions between transmission mode, age and relevance, but given the findings reviewed above, we formulate the following hypotheses, organised -like the result section below- by outcome variable:

Regarding action copying actions present in a participant's input (we call this 'retention'):

1. More retention of actions under Demonstration than under Verbal instruction. In prior

Regarding innovation (production of novel actions not present in a participant's input): studies, verbal instruction plus demonstration conditions sometimes led to increased fidelity with respect to demonstration only. However, this difference was never very large, so we predict that verbal instruction alone will support lower fidelity than observation alone.

2. More retention of Relevant actions than of Irrelevant actions.

3. More action retention in Adults than in Children.

4. More retention of Irrelevant actions (overimitation) in Adults than in Children.

Regarding cumulative improvement of efficiency:

7. A loss of irrelevant actions, but not of relevant actions, over generations (this is our definition of cumulative improvement in efficiency).

8. A more pronounced loss of irrelevant actions under Verbal instruction than under Demonstration (as participants in the latter are expected to retain more actions, see hypothesis H1) 
9. A more pronounced loss of irrelevant actions over generations in Children than in Adults (as adults are better overimitators, see hypothesis $\mathrm{H} 4$ ).

\section{Methods}

The study obtained ethical approval from the Heriot-Watt University's School of Social Sciences ethical committee and it was pre-registered with the Open Science Foundation.

\subsection{Participants}

Seventy adults (31 female; age range 19-32 years, $M=22.94$ years, $S D=3.11$ years) were recruited and tested in public libraries and cafes in Cyprus. Sixty of the participants were assigned to twenty chains (three participants per chain) so that the mean age of every chain was approximately equal, with all chains including male and female participants and the malefemale ratio being similar across chains and conditions. The remaining ten participants formed the adult control group. No compensation was given to the adult participants.

Seventy children (30 female; age range $6-8, M=6.72, S D=0.76$ ) were recruited in Cyprus in summer schools, playgrounds and among the experimenter's personal network. All children had recently finished either their first or second year in primary school. For other studies that use this exact age-group within a condition see e.g. Kumpfer et al. 2002; Patil et al. 2014; Neely et al. 2016. Sixty of the children were assigned to twenty chains (three participants per chain) so that the mean age of every chain was approximately equal, and the male-female ratio was similar across chains and conditions. The remaining ten participants formed the child control group. The children received stickers as compensation for their participation.

Two additional participants were the 'seeds' used to record the stimuli. They were a male adult and a male 9-year old, both native Greek Cypriot speakers.

No data was excluded, as every participant completed the task successfully.

\subsection{Materials}

The apparatus presented to all participants was an adaptation of the opaque box used in previous studies with both children and adults (McGuigan et al. 2007; McGuigan and Whiten 

box were not used (Fig.1).
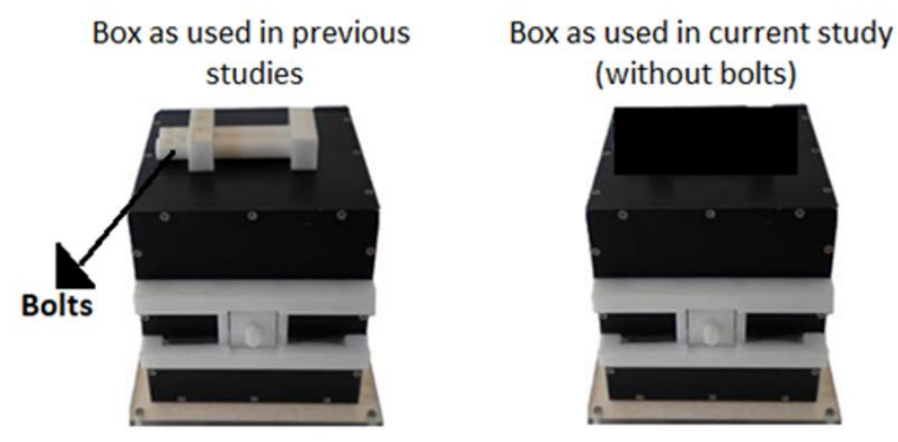

Figure 1. The box used in the experiment.

185 A reward was housed in a tube inside the box. The reward could not be retrieved using a

186 finger; in order to extract it, participants could use a rod with a magnet attached to one end

187 (covered in red tape), and a blue Velcro on the other end. The reward was a metal ball 188 wrapped in Velcro, so it was possible to retrieve it by using either the red or the blue end of 189 the rod.

190

\subsection{Design}

191 Participants were organised into linear transmission chains of three generations, where each participant represented one generation, Fig. 2). We had a total of 40 chains: 20 of children

193 and 20 of adults. The input to each generation was the (video or audio) recorded output of 194 the preceding generation. Generation 0 (the 'seed' participants, see below) provided the 195 initial input for all the chains in the same transmission and age group. The number of 196 generations (the seed plus three generations of participants) was informed by previous 197 studies in which most effects of transmission occur in the first few generations. 


\section{Generation}

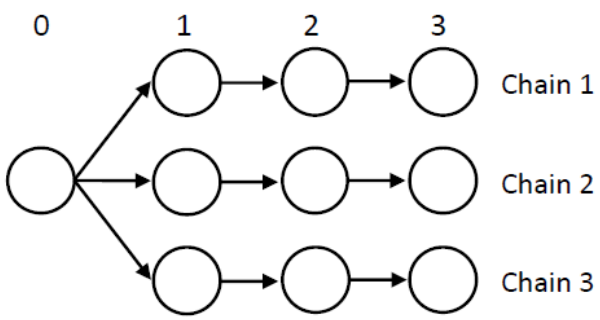

198

199

200

201

202

203

204

205

206

207

208

209

210

211

212

213

214

215

216

217

Figure 2. Linear transmission chain structure. All chains in the same transmission and participant age condition received input from the same Generation 0 'seed' participant.

In addition to participant age, we manipulated action causal relevance (see 2.4 .1 ) and social transmission mode (demonstration or verbal instruction) (see 2.4.2).

\subsection{Procedure}

\subsubsection{Initial action sequences}

The first participant in each chain (the 'seed', or generation 0) was taught one of two different sequences of four actions (Table 1). These sequences were the same for the adult and the child seed and they consisted of two causally relevant actions (Rel), which were necessary for retrieving the reward, and two causally irrelevant (Irrel) actions, that were not necessary for retrieval ${ }^{1}$. Our Relevant actions were used in previous studies using the same apparatus (McGuigan et al. 2007; 2010; 2011). We made up four Irrelevant actions specifically for this study. They were easy to perform and simple enough to remember by adults and children, and took approximately the same amount of time to perform as the Relevant actions, thus balancing the time of participant exposure to both Irrelevant and Relevant actions. Following the two-action approach (Dawson and Foss 1965; Whiten et al. 2016; McGuigan et al. 2007; 2010 ; 2011) a different sequence of actions was the input for half of the chains in each condition (Table 1).

\footnotetext{
${ }^{1}$ At the pre-registration stage, this study intended to include an additional independent variable, namely action familiarity. One half of the causally irrelevant actions at generation 0 were intended to be 'familiar' to the participants and the other half to be 'unfamiliar'. However, we found that participants in the control condition spontaneously performed the 'unfamiliar' actions. This invalidated our criterion to distinguish familiar and unfamiliar actions and, as a consequence, the variable familiarity is not included in this report.
} 
Table 1. Action sequences performed (in the demonstration condition) or verbalised (in the

219 verbal instruction condition) by the seeds.

\begin{tabular}{|c|c|c|}
\hline & Action Performed & Action Verbalisation \\
\hline \multirow{3}{*}{$\begin{array}{l}\text { Sequence } 1 \text { : } \\
\text { for } 10 \text { chains of } \\
\text { children }\end{array}$} & $\begin{array}{l}\text { Tap three times on the side of } \\
\text { the box with the rod (Irrel) }\end{array}$ & $\begin{array}{l}\text { "I tap three times on the side of } \\
\text { the box with the rod" }\end{array}$ \\
\hline & $\begin{array}{l}\text { Swipe rod on the hand three } \\
\text { times (Irrel) }\end{array}$ & $\begin{array}{l}\text { "I swipe the rod on my hand three } \\
\text { times" }\end{array}$ \\
\hline & Lift door with hand (Rel) & "I lift door with my hand" \\
\hline $\begin{array}{l}\text { and } 10 \text { chains of } \\
\text { adults }\end{array}$ & $\begin{array}{l}\text { Insert (red) magnetic side of rod } \\
\text { in door to extract the reward } \\
\text { (Rel) }\end{array}$ & $\begin{array}{l}\text { "I put the red side of the rod in } \\
\text { the door to get the ball" }\end{array}$ \\
\hline \multirow{4}{*}{$\begin{array}{l}\text { Sequence } 2 \text { : } \\
\text { for the other } 10 \\
\text { chains of children } \\
\text { and the other } 10 \\
\text { chains of adults }\end{array}$} & $\begin{array}{l}\text { Pass the rod from hand to hand } \\
\text { three times (Irrel) }\end{array}$ & $\begin{array}{l}\text { "I pass the rod from one hand to } \\
\text { the other three times" }\end{array}$ \\
\hline & $\begin{array}{l}\text { Swipe the rod on the side of the } \\
\text { box three times (Irrel) }\end{array}$ & $\begin{array}{l}\text { "I swipe the rod on the side of the } \\
\text { box up and down three times" }\end{array}$ \\
\hline & Slide door with hand (Rel) & $\begin{array}{l}\text { "I slide the door with my hand to } \\
\text { the side" }\end{array}$ \\
\hline & $\begin{array}{l}\text { Insert (blue) Velcro side of rod in } \\
\text { door to extract the reward (Rel) }\end{array}$ & $\begin{array}{l}\text { "I put the blue side of the rod in } \\
\text { the door to get the ball" }\end{array}$ \\
\hline
\end{tabular}

221 The two seed participants (an adult and a child) were trained by the experimenter to produce

222 the initial action sequences (see Table 1), after obtaining informed consent from the adult 223 and the child's parents. Their live demonstrations (for the Demonstration condition) were 224 video recorded, and the spoken descriptions of their actions (for the Verbal Instruction condition) were audio recorded in separate sessions. For practical reasons, we used recorded information, in line with similar studies (McGuigan et al. 2007; Wood et al. 2012).

\subsubsection{Social learning conditions} Informed consent was obtained from the remaining adult participants and, in the case of children, from their parents. Participants were allocated to one of the following three conditions: 
Demonstration condition: Each participant was offered a seat in front of a laptop and the box (which they could see right next to the laptop). The experimenter told them: "Inside the box there is a ball. The goal is to take the ball out of the box. Before you try, I will show you a video of another participant who took the ball out. The video will play three times. Then, you can try, too". The video recorded actions performed by the previous participant in the chain (or, for generation 1, by the seed) were presented three times on the laptop with the volume muted. Following the task demonstrations, the experimenter invited the participant to "have a go". Their actions were video recorded. Participants were allowed to interact with the box for a maximum of five minutes, or until they retrieved the reward successfully, if this was less than five minutes, or until they became frustrated or refused to continue. All participants were successful in retrieving the reward within time allocated. While the participants were carrying out the task, the experimenter did not interfere.

Verbal Instruction condition: Each participant was offered a seat in front of the box and the laptop and they were told: "Inside the box there is a ball. The goal is to take the ball out of the box. Before you try, I will play for you the [voice] recording of another participant who took the ball out. The recording will play three times. Then, you can try, too". The recorded verbal instructions from the previous participant in the chain (or, for generation 1 , by the seed) were then played three times on a laptop computer ( $\mathrm{mp3}$ player). Following the task demonstrations, the experimenter invited the participant to "have a go", and his or her actions were video recorded. After the participant finished the task, the experimenter gave the following instructions: "I will play for you a video of yourself on my laptop and I want you to watch carefully what you do. Every few seconds, I will pause it. Each time I pause the video, I would like you to tell me what you do in it". To make sure the participant understood the voice-recording procedure, the experimenter asked them if they had any questions. After making sure her instructions were clear, she played for each participant their video and she paused it after every action. She then brought the microphone close to the participant, who described their actions as they saw it. All participants were able to describe their sequence action by action. The experimenter did not guide them as to in which format they should give their descriptions (some of them took a first-person perspective and others a second-person perspective). Their descriptions of their actions were audio recorded, and they were used as the audio input for the next participant in the chain. Participants were allowed to interact 
with the box undisturbed until they retrieved the reward successfully, or after five minutes, as above.

Control condition: The experimenter invited each participant to interact with the box by

265

266 saying: "There is a reward in this box. Can you take it out?". They were allowed to interact with the box undisturbed until they retrieved the reward successfully, or after five minutes, or if they became frustrated or refused to continue (all participants were successful). They were video recorded while they interacted with the box.

In all three conditions, after each participant finished the task, they were thanked and led to a different room to be debriefed. In addition, they were asked, for some of the actions they performed, why they performed it the way they did: "Why did you do x?".

\subsection{Data coding and variables}

The video recording of participants in the Demonstration and the Verbal Instruction conditions were coded by the first author. One more naïve observer -co-author MT- coded random samples $(10 \%)$ of the data for reliability regarding the variables below. Cohen's $\mathrm{K}$ (Landis and Koch 1977) revealed near-perfect inter-coder agreement, $k=.838(p<.000), 95 \%$ Cl $(0.799,0.877)$.

Control condition: Each action participants produced was coded as causally relevant if it contributed to extracting the reward or as causally irrelevant if it did not contribute (see actions produced by control groups in SM.1). All participants in the control group were successful in retrieving the reward from the box. Apart from some attempts to retrieve the reward by inserting their finger in the door, there were no causally irrelevant actions.

Transmission chains: For coding and analysis purposes, our units were action types in a participant's input and/or output. If an action type was present in a participant's input or output, the type was included. Action tokens allocated to an action type included exact replicas, slight modifications and variations in the number of times an action is produced (e.g. swiping the rod on the hand 3 or 4 or more times were all counted as the same action type).

For each action type produced by a participant, we coded: Participant ID; participant Age group; Chain; Generation; Action type (all action types in our data are listed in SM.2); whether 
290

291

292

293

294

295

296

297

298

299

300

301

302

303

304

305

306

307

308

309

310

311

312

313

314

315

316

317

318

the action was present in the participant's input (witnessed) or not; whether the action was produced by the participant or not; the transmission mode in which the action was produced (Demonstration or Verbal instruction); and action causal Relevance (whether the action contributed to extracting the reward or not). From this information, we extracted three outcome variables:

(i) Action retention reflects whether an action produced by the previous participant (or by the seed in the case of generation 1) was reproduced by the current participant. This binary variable was only applied to actions that were present in a participant's input (if it was not present, then retention $=\mathrm{NA}$ ). If the action was also present in the participant's output, then retention $=1$, otherwise retention $=0$. (Note that for the purposes of action Retention, variable Generation was not taken into account: rather, for each action in any participant's input, we coded whether they retained it their output or not.)

(ii) Action innovation: A variable with value 1 if a participant produced an action that had not been present in their input (an innovative action). Example innovative actions include throwing the rod in the air and catching it and tapping on the box with the hands (see full list in SM.2).

(iii) Cumulative improvement of efficiency. This variable was operationalised as a decrease in the number of irrelevant actions in participants' outputs over generations. Eliminating irrelevant actions decreases the time to completion of the task, while not detracting from achieving the goal of extracting the reward from the box. Therefore, losing irrelevant actions increases efficiency. For this analysis, actions produced at Generations 1,2 and 3 were included (but not actions produced at Generation 0 ). (This is different from action retention and innovation. Here, we consider the proportion of irrelevant actions out of all the actions produced by participants, regardless of whether they are copied or innovated.)

\section{Results}

Table 2 shows, for each generation, the number of action tokens in participants' input, which can be broken down into number retained plus number lost. It also shows the number of actions produced by participants which equals the sum of actions retained plus 
319

320

321

322

323

324

325

326

327

328

329

330

331

332

333

334

335

336

337

338

339

340

341

342

actions innovated. (The output of generation 3 was not actually used as input, as our chains were 3 generations long.) Overall, actions decreased generations from the 160 actions produced by the seed (generation 0 ) to the 108 actions produced by generation 3 $\left(\chi^{2}(3)=11.68, p=0.009\right)$.

Table 2. The fate of actions by generation.

\begin{tabular}{crrrrr}
\hline Generation & In input & Retained & Lost & Innovated & In output \\
\hline G1 & 160 & 107 & 53 & 27 & 134 \\
G2 & 133 & 77 & 56 & 42 & 119 \\
G3 & 119 & 88 & 31 & 20 & 108 \\
\hline Total & 412 & 272 & 140 & 89 & 361 \\
\hline
\end{tabular}

The results reported below are connected to hypotheses as numbered in the Introduction section.

\section{1 Action retention}

We used glmer from the lme 4 package (Bates, Maechler \& Bolker, 2012; Bates et al. 2015) and the bobyqa optimiser in $R^{2}$ ( $R$ Core Team, 2012) to estimate a mixed effects logistic regression model. The binary outcome variable was action Retention; the fixed effects were all binary: transmission mode (verbal instruction/demonstration), participant age (adult/child) and action relevance (causally relevant/irrelevant). We included random intercepts for participant and action (as actions varied in the degree they were retained, see 3.2 below). We also included the interactions of the three fixed effects, as the model with interactions was significantly better than the one which did not $(\chi 2=13.67, d f=4, p<0.01)$. The function anova () found that the full model (RETENTION Causal Relevance * Transmission Mode * Age + (1 | Participant) + (1 | Action)) was significantly better than a null model including only the random effects $(\chi 2=18.77, \mathrm{df}=7, \mathrm{p}<0.01)$. Table 3 summarises the full model.

\footnotetext{
${ }^{2}$ For analysis code in R see SM.3.
} 
Table 3. Summary of the logistic regression model. Estimates are on the logit scale.

\begin{tabular}{|c|c|c|c|c|c|}
\hline \multicolumn{6}{|c|}{ Model: Causal Relevance * Transmission Mode * Age + (1 | Participant) + (1 | Action) } \\
\hline & Est. & SE & $\mathbf{C l}$ & $z$ & $p$ \\
\hline Relevance (Rel) & 1.67 & 0.811 & $(0.08,3.26)$ & 2.059 & $0.040^{*}$ \\
\hline Transmission (Dem) & 1.09 & 0.735 & $(-0.35,2.53)$ & 1.486 & 0.137 \\
\hline Age (Child) & 1.57 & 0.694 & $(0.20,2.92)$ & 2.255 & $0.024^{*}$ \\
\hline Relevance (Rel) x Transmission (Demo) & -0.01 & 0.853 & $(-1.68,1.67)$ & -0.006 & 0.995 \\
\hline Relevance (Rel) x Age (Child) & -1.48 & 0.798 & $(-3.05,0.08)$ & -1.858 & 0.063 \\
\hline Transmission (Demo) x Age (Child) (Children) & -2.36 & 0.979 & $(-4.28,-0.44)$ & -2.411 & $0.016^{*}$ \\
\hline Relev. (Rel) x Transm. (Demo) x Age (Chi) & 2.45 & 1.178 & $(0.14,4.76)$ & 2.080 & $0.037^{*}$ \\
\hline
\end{tabular}

Marginal $R^{2}: 0.14$, Conditional $R^{2}: 0.47$

Regarding main effects, the model does not support hypothesis $\mathrm{H} 1$, which predicted more actions retained under Demonstration than Verbal instruction. The model supports $\mathrm{H} 2$, as it finds a significant main effects of action Relevance on Retention, with participants being more likely to retain causally Relevant than Irrelevant actions overall (Fig. 3A). And it finds the opposite of what was predicted in $\mathrm{H} 3$. In our data, children retain significantly more actions than Adults overall (Fig. 3B).

$3 A$

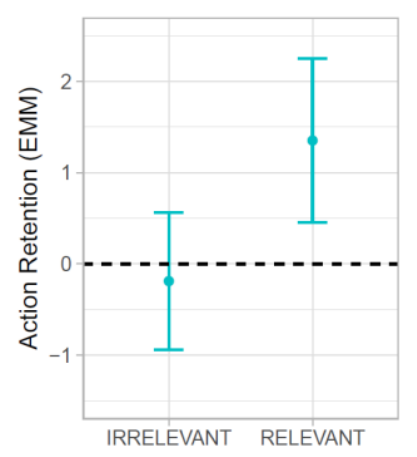

3B

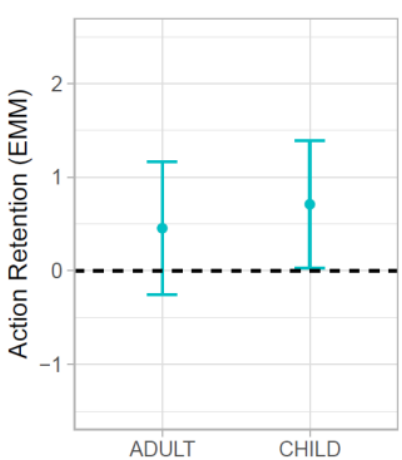

$3 C$

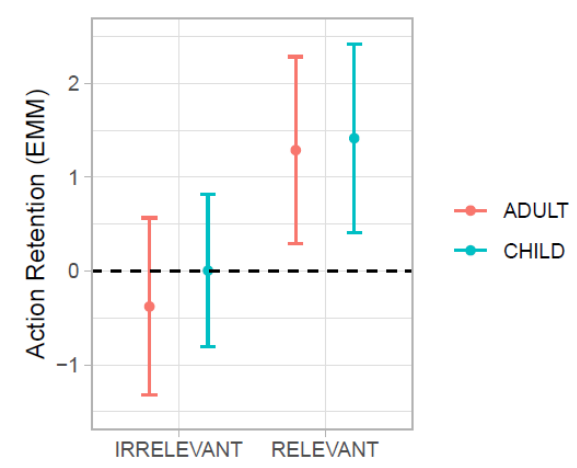

Figure 3. Estimated marginal means (Log odds) of action Retention illustrating the effects related to hypotheses $\mathrm{H} 2, \mathrm{H} 3$ and $\mathrm{H} 4$.

355 Hypothesis H4 predicted more Retention of Irrelevant actions (overimitation) in Adults than in Children; the interaction in the model not quite significant. As was the case with $\mathrm{H} 3$, however, the difference in our data is in the opposite direction as was predicted. We find more overimitation in children than adults (Fig. 3C). 
359 We also find a significant 3-way interaction between the three predictors (Table 3). Following 360 the marginality principle (Nelder, 1977), we will focus on this interaction. Figure 4 shows the 361 estimated marginal means of action Retention for each condition combination and Table 4 the significant pairwise contrasts (the full table of contrasts is in SM.4).

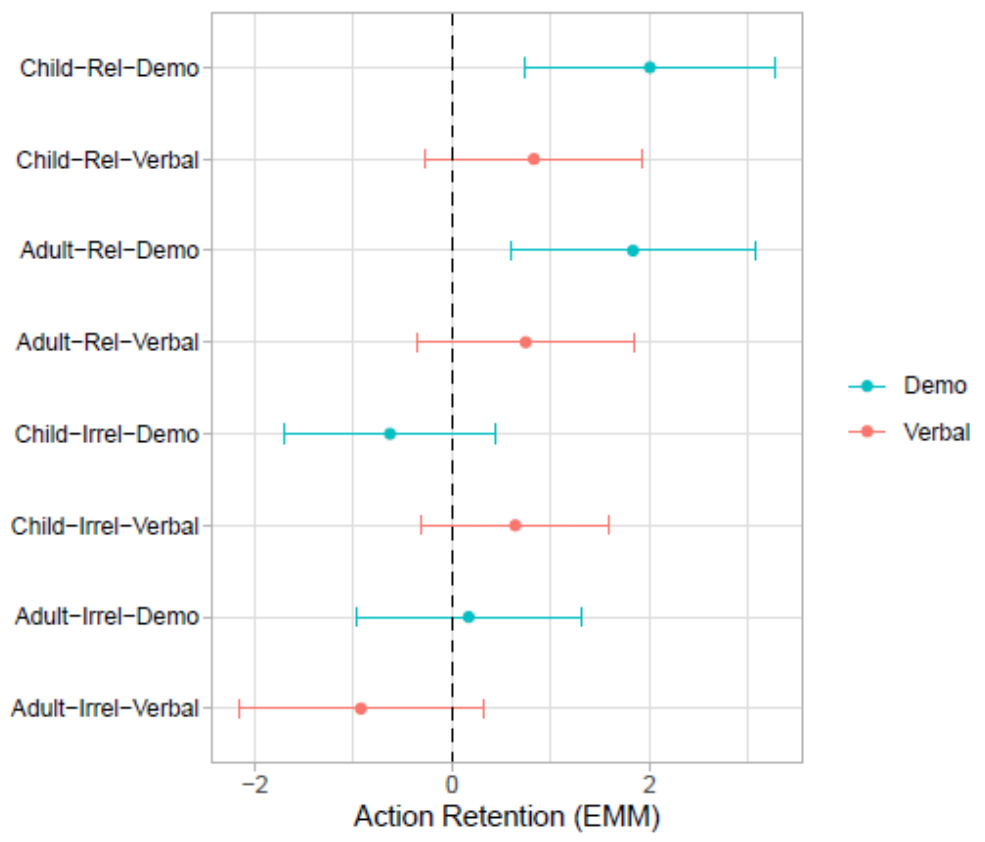

364 Figure 4. Estimated marginal means (Log odds) of action Retention for all combinations of the three predictors:

Table 4. Significant pairwise contrasts out of the 28 contrasts (full table in SM.4).

\begin{tabular}{crrrr}
\hline Contrast & $\begin{array}{r}\text { Estimate } \\
\text { (Log-odds } \\
\text { ratio) }\end{array}$ & SE & z-ratio & $\begin{array}{r}p \text {-value } \\
\text { (Bonferroni } \\
\text { corrected) }\end{array}$ \\
\hline Adult-Irrel-Verbal - Adult-Rel-Demo & -2.758 & 0.919 & -3.000 & 0.024 \\
Adult-Irrel- Verbal - Child-Rel-Demo & -2.929 & 0.926 & -3.165 & 0.022 \\
Child-Irrel-Demo - Adult-Rel- Demo & -2.463 & 0.843 & -2.923 & 0.024 \\
Child-Irrel- Demo - Child-Rel-Demo & -2.634 & 0.795 & -3.314 & 0.022 \\
\hline
\end{tabular}

369 Figure 4 illustrates the higher action retention of Relevant (top four lines) than irrelevant 370 actions (bottom four lines). It also shows that EMMs for children are somewhat higher than

371 for adults in matching conditions. And the overall pattern whereby for each age group and 372 relevance condition, more actions are retained under Demonstration (in blue in Fig. 4) than 
under Verbal instruction (in red), except for children retention of irrelevant actions

374 (overimitation), where the pattern is reversed.

375

376

377

378

379

380

381

382

383

384

385

386

387

388

389

390

391

392

393

394

395

396

\section{2 Action innovation}

Actions produced by a participant that had not been present in their input were counted as innovative actions. At each generation, $20 \%, 35 \%$ and $19 \%$, respectively, of all action tokens produced by a participant were innovations (Table 2). A total of 89 action tokens (31 types) were produced by participants in transmission chains who did not have those actions in their input. The most common innovations were the alternative methods to open the door or extract the reward, in particular lifting the door open after seeing/hearing that the previous participant had slid it open and using the magnetic (red) end of the rod after having seen/heard the previous participant used the velcro (blue) end (Figure 5).

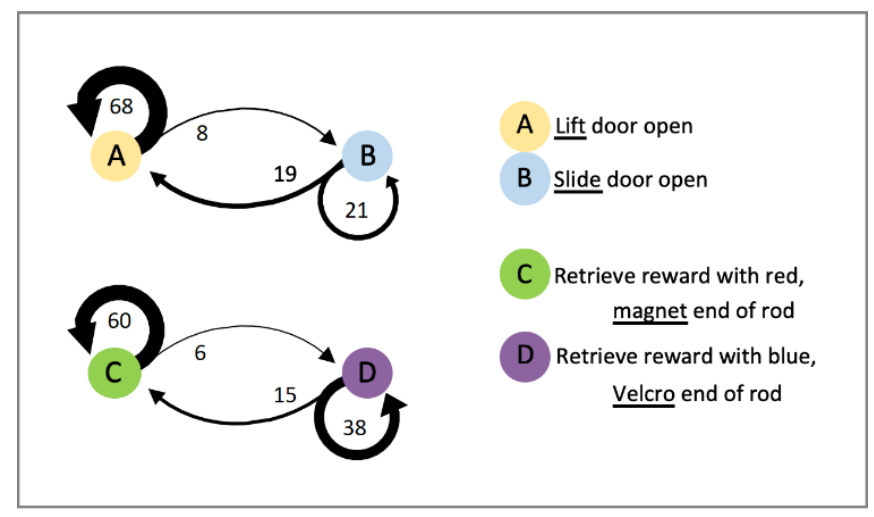

Figure 5. The fate of actions, illustrating innovation. For each of the four causally-relevant actions, the extent to which they were retained in their input (arrow to itself) or they were lost, and replaced in the output by the alternative action for the same function (arrow to the other action). E.g., of the 40 participants who had action $B$ in their input, 21 retained it in their output and 19 produced the alternative method A. See also SM. 4 for the fate of all action types.

To test hypotheses $\mathrm{H} 5$ and $\mathrm{H6}$, we used Chi-squared tests of independence to test the effects of participant age (adult/child), action relevance (causally relevant/irrelevant) and transmission mode (verbal instruction/demonstration) on Innovation. Regarding H5, we found a significant effect of Transmission condition $\left(\chi^{2}(1)=4.67, p<0.05\right)$, with more Innovation in the Verbal Instruction ( $N=57$ ) than the Demonstration condition ( $N=32)$ (Fig. 6). Regarding H6, we found no effect of Relevance (H6) (Relevant $\mathrm{N}=56$, Irrelevant $\mathrm{N}=33$ ). There was, additionally, no difference in Innovation by Age (adults $\mathrm{N}=39$, children $\mathrm{N}=50$ ). 


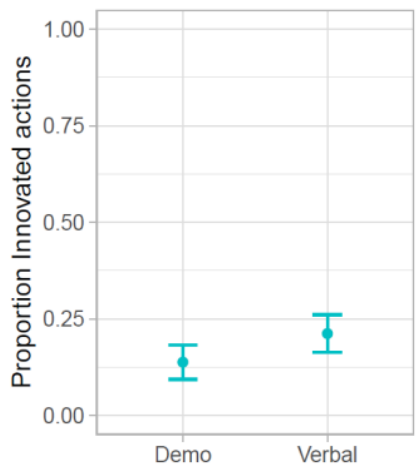

Figure 6. Proportion of actions that were innovated (introduced by participants in their output) by

Transmission condition.

400

401

\subsection{Cumulative improvement in efficiency}

To test hypotheses 7-9, we used glmer from the lme 4 package (Bates, Maechler \& Bolker, 2012; Bates et al. 2015) and the bobyqa optimiser in R (R Core Team, 2012) to estimate a mixed effects logistic regression model. The binary outcome variable was action Relevance. We included Generation as a continuous fixed effect to test for cumulative improvement (a specific decrease of Irrelevant actions over Generations), and also three interactions: Generation by Transmission (to test the effect of Transmission on specific change in Irrelevant actions over Generations); Generation by participant Age (to test the effect of participant Age on specific change in Irrelevant actions over Generations); and Generation by Transmission mode by participant Age (to test for the interaction between Transmission and participant Age on specific change in Irrelevant actions over generations). We included a random intercept for chain. The full model (RELEVANCE Generation + Generation:Transmission Mode + Generation:Participant Age + Generation:Transmission Mode:Participant Age + (1 / Chain)) was tested against the null model, which contained only the random effect, using the anova ( ) function and the difference was found to be significant

The full model (RELEVANCE Generation + Generation:Transmission Mode + Generation:Participant Age + Generation:Transmission Mode:Participant Age + (1 / Chain)) was tested against the null model, which contained only the random effect using the anova ( ) function and was found to be significantly better $(\chi 2=21.13, d f=4, p<0.001)$. Table 5 summarises the full model. 


\begin{tabular}{|c|c|c|c|c|c|}
\hline \multicolumn{6}{|c|}{$\begin{array}{r}\text { Model: Generation + (Generation x Age })+(\text { Generation x Transmissic } \\
\text { Transmission Mode } \times \text { Age })+(1 \mid \text { Chain })\end{array}$} \\
\hline & Est. & SE & $\mathbf{C l}$ & $\bar{z}$ & $p$ \\
\hline Generation & 0.76 & 0.23 & $(0.32,1.20)$ & 3.37 & $<0.001$ \\
\hline Gen. x Age (Child) & -0.69 & 0.19 & $(-1.07,-0.31)$ & -3.6 & $<0.001$ \\
\hline Gen. x Transmission (Demo) & -0.38 & 0.2 & $(-0.78,0.01)$ & -1.9 & 0.06 \\
\hline Gen. x Age (Child) x Transmission (Demo) & 0.77 & 0.26 & $(0.26,1.28)$ & 2.97 & 0.003 \\
\hline
\end{tabular}

Marginal $R^{2}: 0.1$, Conditional $R^{2}: 0.1$

422 These results support H7 that Irrelevant actions, but not Relevant ones, would decrease over

423 generations (Fig. 7). This specific loss of Irrelevant actions over generations is our definition 424 of cumulative improvement in efficiency.

425

426

427 Results do not support the stronger cumulative improvement under Verbal instruction than 428

429 430 431

Figure 7. Counts of causally Relevant and Irrelevant actions produced at each Generation. under Demonstration predicted by $\mathrm{H} 8$.

Hypothesis H9 predicted a stronger cumulative improvement in Children than Adults. However, we find the opposite effect: a significant interaction between Generation and participant Age, whereby Adults lose more irrelevant actions than Children (Fig. 8).

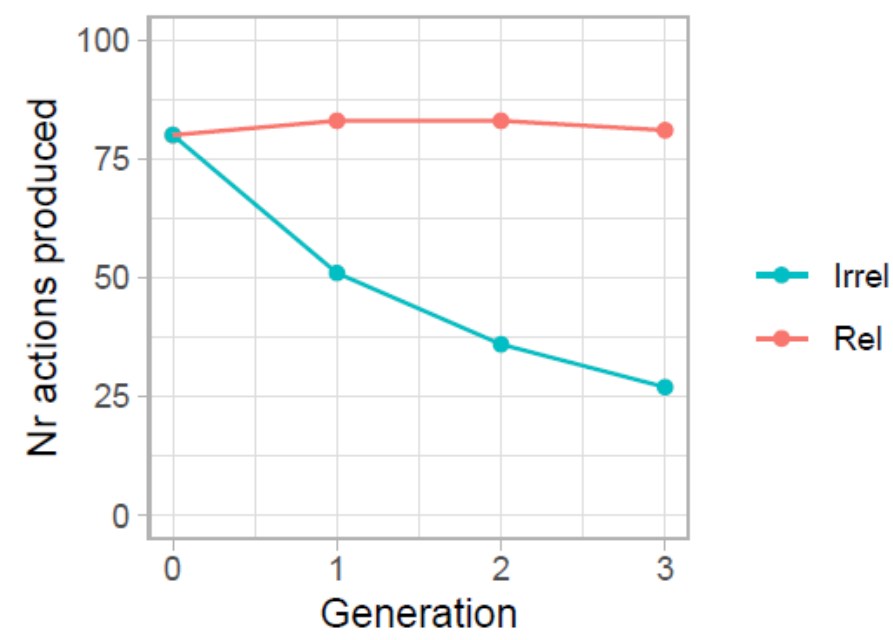




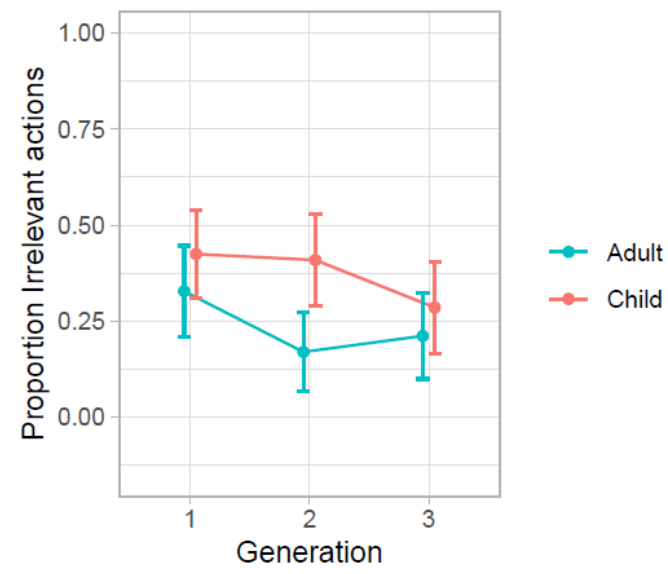

434 Independently of the hypothesis presented in the introduction, we found a significant 3-way 435 interaction between Generation, Transmission mode and participant Age. This is illustrated in 436 Fig. 9 (as the proportions of irrelevant actions) and in Fig. 10 as the Estimated Marginal Means 437 for Cumulative Improvement (in other words, the interaction between Generation and action 438 Relevance).

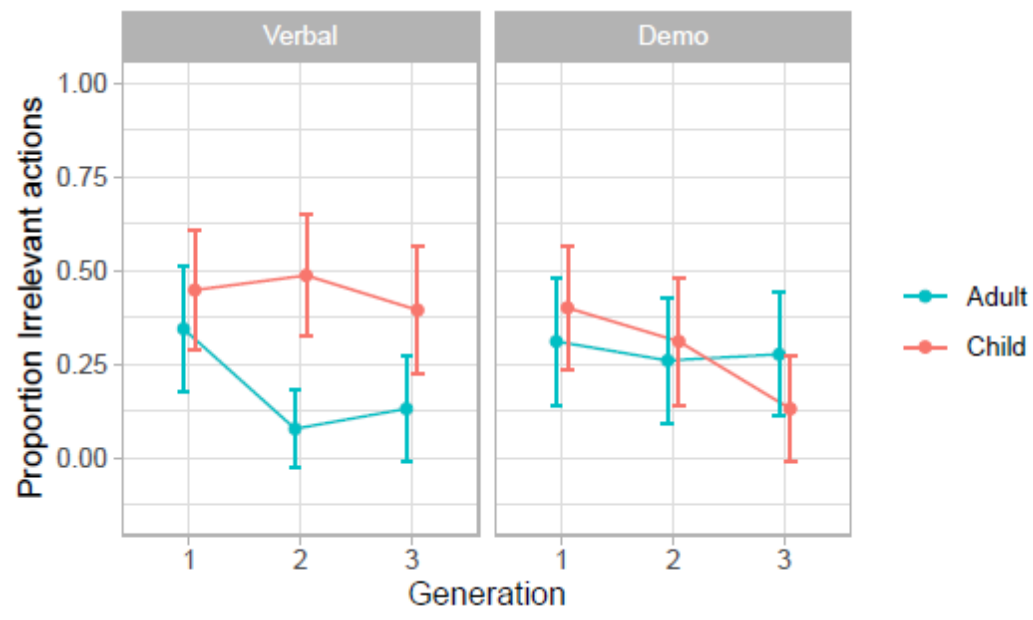

440 Figure 9. Proportion of all produced actions that were Irrelevant, by Transmission condition and participant Age. 


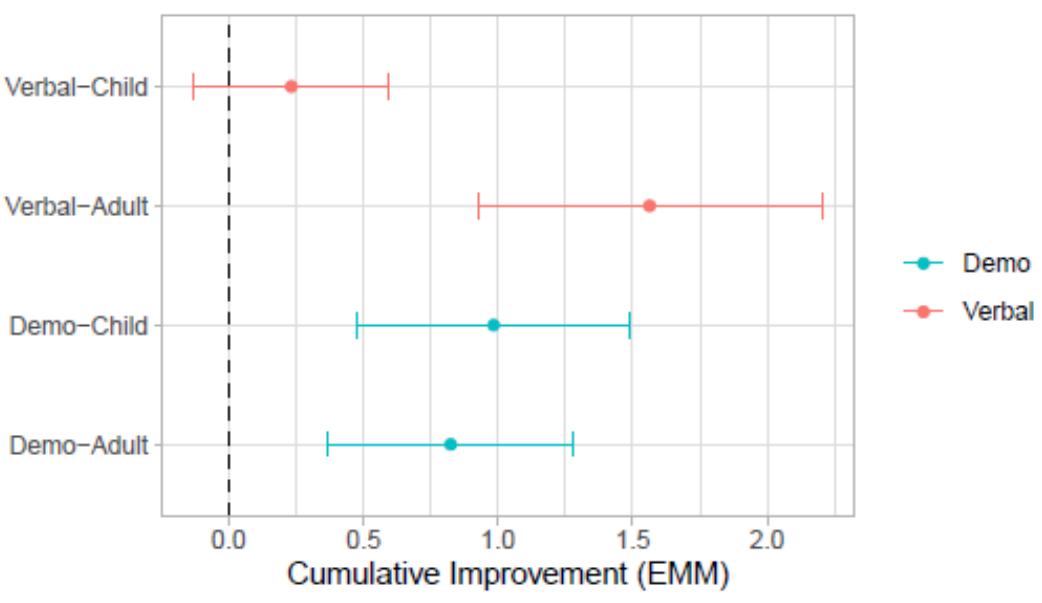

442

Figure 10. Estimated marginal means of Cumulative Improvement in the different conditions of participant Age and Transmission condition.

445 The estimated marginal means in Fig. 10 represent differences in how Relevant and Irrelevant 446 actions change over generations. The positive EMMs indicates that, in all cases, Irrelevant 447 actions disappear faster than Relevant actions (Fig. 9). While in the Demonstration condition 448 there is no difference between Children and Adults, in the Verbal condition Adults lose 449 significantly more actions over generations than Children $(\log O R=1.331, S E=0.369$, z450 ratio=3.604). In addition, Children lose significantly more actions over generations under 451 Demonstration than under Verbal transmission ( $\log \mathrm{OR}=-0.751, \mathrm{SE}-0.398$, z-ratio=-2.439). (See 452 all pairwise contrasts in SM.5).

453

\section{Discussion}

455 The main objective of the current study was directly and systematically to compare for the 456 first time the effects of purely language-based transmission (which did not allow gesture or 457 other visual cues) against visual demonstration (the transmission modality used in most 458 previous studies of social learning) on social learning and cultural evolution. We analysed the extent to which actions were retained by participants, how much innovation participants introduced and how the efficiency of action sequences cumulatively evolved over three generations. Our sequences included causally relevant and irrelevant actions to test the effects of transmission mode on imitation and overimitation, respectively, and we included adult and 6-8-year-old participants to explore developmental effects. 
Both our adult and child participants retained many of the causally irrelevant actions present in their input. This contributes to the established body of evidence of overimitation in humans. In the control condition, participants produced a few causally irrelevant actions, but they were in all likelihood exploratory, and could have turned out to be causally relevant (e.g. try to extract the reward with their fingers). In the transmission chains, in contrast, it was obvious that at least some actions were not necessary, but they were nevertheless retained, indicating that participants were much more likely to produce manifestly superfluous actions in the context of social learning than asocial learning.

We found support for our hypotheses $\mathrm{H} 2$ and $\mathrm{H} 7$, namely more retention of relevant than irrelevant actions, in line with previous studies (e.g. Flynn 2008; McGuigan and Whiten 2009; McGuigan and Graham 2009; McGuigan et al. 2011), and the cumulative specific loss of irrelevant actions over generations. However, our results did not support $\mathrm{H} 4$ and $\mathrm{H} 9$, which predicted more overimitation by adults than children.

We did not find support for more action retention under demonstration than under verbal instruction overall. However, this is the case for imitation and overimitation by adults, and imitation by children. Interestingly, the exception was overimitation by children, which was higher under verbal transmission than under demonstration (Fig. 4). In other words, when presented with causally relevant actions, all our participants imitated more when the actions were demonstrated visually than when they were conveyed verbally, that is, they imitated more when they saw the actions than when they were told about them. (When they did not imitate a causally relevant action, they innovated: they replaced it with an unobserved action that would allow them to complete the task). When presented with causally irrelevant actions, adults did the same, they copied more what they saw than what they were told about. However, children behaved in the opposite way - they reproduced fewer of the causally irrelevant actions they saw than of the actions they were told about. This suggests that, when it comes to imitation, verbal transmission increases the likelihood that participants of any age ignore, forget or misunderstand (or perhaps be more inspired to be creative) compared to visual demonstration. The same is the case with overimitation in adults. When it comes to overimitation in children, in stark contrast, verbal transmission contributes to transmission fidelity. 
Even though the majority of actions were retained, many participants introduced innovative

495

496

497

498

499

500

501

502

503

504

505

506

507

508

509

510

511

512

513

514

515

516

517

518

519

520

521

522

523

524 actions that were not present in their input. For instance, a participant tilted the box towards them so the ball rolled out of the door and therefore it was not necessary to use the rod; another one pushed the door open with the rod, instead with their hand. When asked why they had replaced actions in their input with new ones, some participants responded that they could not remember the exact actions in the input, or that they thought that was the way to do it. Most of them, however, were not sure why they had innovated.

More innovation occurred under verbal instruction than under demonstration. A possible reason for the lower transmission fidelity (less retention and more innovation) observed under verbal instruction is the visual absence of the model in our audio recorded instructions. This possibility can be tested by conducting a similar study in which video input is presented in both conditions, but in one the model demonstrates and in the other narrates the action sequence. Alternatively, lower fidelity under the verbal instruction condition may be due to language underspecification (e.g. Frisson 2009). For a physical task such as extracting a reward from a box, the meaning conveyed by verbal signals is less detailed and more ambiguous than the meaning conveyed by visual demonstration. The meaning of a verbal signal (in our case, an instruction) is shaped by the listener's prior knowledge and by the context. Suppose a participant hears 'swipe the rod three times on the side of the box'. In order to carry out the instruction, they have to access their own meaning for swipe, rod, three, etc. While some signals are unambiguous for most speakers (e.g. three times) others, such as swipe, can be interpreted in multiple ways (e.g. swipe upwards, downwards, from left to right etc; holding the rod with two fingers or with the whole hand, etc.). Demonstration provides a level of detail that significantly narrows down interpretation. This could explain some of our results, but not the higher retention of irrelevant actions (overimitation) by children under verbal transmission. Indeed, children retained relevant and irrelevant actions to the same degree when they were verbally instructed (compare $2^{\text {nd }}$ and $6^{\text {th }}$ lines in Fig. 4). This exception may be explained by links between language and normativity.

Overimitation in children has been related to a psychological tendency to participate in rituals -conventional, causally opaque action sequences- which, in turn, facilitate social affiliation (Wen et al. 2016), and to normativity, the belief that it is somehow obligatory to copy behaviour faithfully (Keupp et al. 2013), which may promote cooperation (Rakoczy et al. 
525 2009). Children tend to assume that socially learned actions are normative to the extent that 526 they denounce others who perform modified versions of the actions. They display normative 527 behaviour after adult models give normative instructions (Casler et al. 2009; Rakoczy et al. 528 2008), and even when the adult's language is not explicitly normative (Schmidt et al. 2011). 529 Language elicits normativity and maintains rituals (e.g., saying "I will show you how it works" 530 implies the behaviour shown is the norm or a ritual), and this may be the explanation why our children overimitate more under verbal instruction. This hypothesis could be tested with direct comparisons of the effects of normative language, non-normative language and no language (e.g., demonstration). If it were supported, it would speak to the question of language origins, suggesting that language may have coevolved with social transmission fidelity. By eliciting normative and ritual behaviour in childhood, language would support the faithful transmission of behavioural patterns (even patterns whose function is not understood), which is required for cumulative cultural evolution (Tamariz 2019), early in life. The fact that overimitation is facilitated by verbal transmission in children, but not in adults indicates that the normative function of language may change over the lifetime. Studying language- and demonstration-mediated social transmission in a range of ages, from early childhood through adolescence and adulthood to old age, would tell us when and how these changes happen. These studies would provide data about the contribution of individuals at different life stages to social transmission, which could in turn inform models of cultural evolutionary dynamics in populations with different age distributions.

Our results for the demonstration condition show no difference between adult and child overimitation levels, in line with some previous studies that also used demonstration (Whiten et al. 2016), but different from others, which found an increase in overimitation with age (e.g., McGuigan et al. 2011; McGuigan 2012). As discussed above, we found more overimitation specifically by children in our verbal condition. This might be due to qualitative differences between the verbal instructions produced by children and adults. Children's utterances could be less complex or more straightforward than adults', and they could facilitate production of causally irrelevant actions. Or children's language might be more goal-oriented, and therefore more suited to instructions about causally relevant actions. But if that were the case, we would not have the distinction between causally relevant and irrelevant actions; instead, we would expect children's retention of actions to be higher in all verbal instruction conditions - 
556 for both relevant and irrelevant actions. Despite that, in order to check whether the

557 differences in children's and adults' speech affected their likelihood of retaining an action in

558 the verbal instruction condition, we transcribed children's and adults' descriptions of the 559 same sequences of actions and compared their narrative styles: which verbs and nouns they 560 used and in what numbers, in which person the verbs were, the length of the descriptions 561 and the number and length of their pauses. We did not find any substantial differences that 562 might account for increased retention in children, so we may conclude that it was the mode 563 of transmission that affected differential retention in children and adults. The country in 564 which the experiment took place could also have contributed to age related differences in 565 overimitation. Cross-cultural differences can affect children's overimitation (Berl and Hewlett 2015; Clegg and Legare 2016), and the primary education system in Cyprus, more teachercentred than other western systems (Papanastasiou 2002), could have biased our child participants to be more compliant with verbal instructions. In Cyprus, teachers traditionally provide instructions and teach children to follow them from a young age. Many of our adult participants were either current students or (recent) graduates of European universities, where they were most probably exposed to more student-centred educational systems. We cannot, however, from the results of our study, ascertain what accounts for the developmental decrease in overimitation under verbal instruction: Did Cypriot adults overimitate less or did Cypriot children overimitate that much more? Studies of languagemediated social transmission with children and adults from countries with different educational styles could shed more light on this question and, by extension, on the effects of the educational system on social learning and cultural evolution.

Our findings, therefore, suggest that verbal transmission generally reduces fidelity compared to demonstration, except in the case of overimitation by children, which is facilitated by verbal transmission. This result is mirrored in the analysis of cumulative change over generations, which is simply a different way to approach the same data that takes generations into account. We operationalised cumulative improvement in efficiency as a reduction in the proportion of causally irrelevant elements produced in the action sequence leading to the extraction of the reward from the box. We found that adults lost more irrelevant actions over generations than children, but this was significant only with verbal instructions. Children, on the other hand, lost more irrelevant actions in the demonstration condition (Fig. 10). 
587

588

589

590

591

592

593

594

595

596

597

598

599

600

601

602

603

604

605

606

607

608

609

610

611

612

613

614

615

616

617

We might equate our measure of cumulative increase in efficiency with cumulative cultural evolution and conclude, first, that the relatively higher cumulative efficiency gains under demonstration in adults is evidence that verbal instruction reduces cumulative culture and second, that, specifically, adults under verbal instruction show the strongest cumulative culture. However, we interpret these results in the opposite way. Our metric of cumulative improvement is based on the principle that eliminating irrelevant actions improves the efficiency of the task at hand. If we take a longer-term evolutionary perspective, we see that this operationalisation actually runs against cumulative cultural evolution: Cumulative cultural evolution results in cultural traits that could not have been invented by a single individual, and these are passed on from generation to generation (Tomasello 1990; Tennie et al. 2009). Such traits can be complex and opaque, so their causal relevance will not be immediately obvious to the learner. The retention of opaque traits cannot be achieved by imitators, who are focused on ends, or goals. Instead, it requires copiers who focus on means, i.e., on the form of actions, such as overimitators. In our study, children failed to improve the efficiency of action sequences over generations under verbal instruction (Figs. 9 and 10). This is because they did not lose (i.e., they retained) causally irrelevant actions (see discussion of the results for retention above). We can therefore conclude that verbal instruction is, specifically in childen, a conservative transmission modality that promotes the faithful retention of causally irrelevant (and potentially opaquely functional) behaviour.

Putt et al. (2014) also found higher transmission fidelity in the form of more precise reproduction of actions when verbal instruction was added to demonstration of stone-tool manufacture, a highly complex skill. Their participants, however, were adults. Tamariz (2019) argues that the essential component of cumulative culture is the replication of actions, regardless of whether their function is understood or not, by naïve learners. Putt et al.'s (2014) adult learners were naïve to their complex task. Our child participants were also arguably naïve to the task of extracting a reward from a strange-looking box. Our adult participants were also naïve to this task, but to a lesser extent than the children. Children are still capable of magical thinking and entertaining supernatural beliefs (Wooley 1997; Subbotsky 2004), which makes them much more naïve, bias-free, open-minded when faced with our causally irrelevant actions. Adults, in contrast, will more readily see the causally irrelevant actions for what they are and, under a rational efficiency bias, will be less likely to 

copy them. Therefore, we can say that both in Putt et al. (2014) and our study, language increased transmission fidelity by naïve (but not by experienced) participants. We resume the

620 idea mentioned above and put forward the hypothesis that language selectively increases blanket copying (McGuigan et al. 2010) or action replication (Tamariz 2019) by naïve learners, and that it enhances not only overimitation, but also the reproduction of the precise structure, or style, of causally relevant actions. (These, in turn, support cumulative cultural evolution which, we should add to close the circle, in turn increases human fitness, e.g. Mesoudi and Thornton 2018). This hypothesis can be tested by comparing verbal and demonstration-based transmission using tasks that are either truly novel or familiar for the participants.

628

The action sequences in this study had a very specific goal, namely to extract a reward from a box. Other experiments exploring verbal transmission versus demonstration used similarly physical or technological goals such as manufacturing stone tools (Onhuma et al. 1997; Morgan et al. 2015; Putt et al. 2014), baskets (Zwirner and Thornton 2015), etc. Cultural traditions, however, are hugely diverse, and transmission and innovation operate differently across cultural domains (Tamariz et al. 2016). We do not know whether our findings apply to other cultural traits such as complex behaviour, ideas, beliefs, values etc. Extensions of the current study may help reveal how transmission fidelity and innovation in children and adults operate under language- and demonstration-based transmission for a variety of cultural traditions.

In conclusion, this study has shed light on how language shapes the operation of the dual engines of cultural evolution -copying and innovation (Legare and Nielsen 2015). We have found that, compared to demonstration, verbal transmission increases innovation; reduces overimitation in adults; and, most interestingly, facilitates overimitation in children.

642 Moreover, under verbal instruction, children overimitate more than adults -the first experimental instance in which this is the case-, suggesting a developmental role of languagemediated social transmission. The evolutionary consequences of increased overimitation in children at the population level and in the long term include higher retention of opaque actions over generations, which suggests a novel explanation for language origin: Language might have evolved in part because it facilitates the faithful transmission of opaque behaviour 
to early learners and, thus, it supports cumulative cultural evolution. This corollary should be

649 further explored with especially designed studies.

650

651

652

653

654

655

656

657

658

659

660

661

662

663

664

665

666

667

668

669

670

671

672

673

674

675

676

677

678

679

680

681

682

\section{References}

Bates D, Maechler M, Bolker B (2015) Walker., S. Fitting linear mixed-effects models using Ime4. J Stat Softw 67:1-48

Bates DM M. \& Bolker, B. Ime4: linear mixed-effects models using 'Eigen'and S4. R package version 1.1-11 (2012)

Berl REW, Hewlett BS (2015) Cultural Variation in the Use of Overimitation by the Aka and Ngandu of the Congo Basin. PLoS ONE 10(3): e0120180. https://doi.org/10.1371/journal.pone.0120180

Boyd R, Richerson P (1996) Why culture is common, but cultural evolution is rare. Proceedings-British Academy. 88:77-94.

Caldwell C, Millen A (2009) Social Learning Mechanisms and Cumulative Cultural Evolution: is imitation necessary?. Psychological Science 20:1478-1483. https://doi.org/10.1111/i.1467-9280.2009.02469.x

Call J, Carpenter M, Tomasello M (2004) Copying results and copying actions in the process of social learning: chimpanzees (Pan troglodytes) and human children (Homo sapiens). Animal Cognition 8:151-163. https://doi.org/10.1007/s10071-004-0237-8

Casler K, Terziyan T, Greene K (2009) Toddlers view artifact function normatively. Cognitive Development 24:240-247. https://doi.org/10.1016/j.cogdev.2009.03.005

Clegg J, Legare C (2015) Instrumental and Conventional Interpretations of Behavior Are Associated With Distinct Outcomes in Early Childhood. Child Development 87:527542. https://doi.org/10.1111/cdev.12472

Clegg J, Legare C (2016) A cross-cultural comparison of children's imitative flexibility. Developmental Psychology 52:1435-1444. https://doi.org/10.1037/dev0000131

Clegg J, Legare C (2017) Parents scaffold flexible imitation during early childhood. Journal of Experimental Child Psychology 153:1-14. https://doi.org/10.1016/j.jecp.2016.08.004

Dawson B, Foss B (1965) Observational learning in budgerigars. Animal Behaviour 13:470-474. https://doi.org/10.1016/0003-3472(65)90108-9

Dean L, Kendal R, Schapiro S et al. (2012) Identification of the Social and Cognitive Processes Underlying Human Cumulative Culture. Science 335:1114-1118. https://doi.org/10.1126/science.1213969

Flynn E (2008) Investigating children as cultural magnets: do young children transmit redundant information along diffusion chains?. Philosophical Transactions of the 
683

684

685

686

687

688

689

690

691

692

693

694

695

696

697

698

699

700

701

702

703

704

705

706

707

708

709

710

711

712

713

714

715

716

717

718

719

720

721

722

Royal Society B: Biological Sciences

363:3541-3551. https://doi.org/10.1098/rstb.2008.0136

Flynn E, Smith K, Kemmelmeier M, Kühnen U (2012) Investigating the Mechanisms of Cultural Acquisition. Social Psychology 43:185-195. https://doi.org/10.1027/1864$\underline{9335 / a 000119}$

Frisson S (2009) Semantic Underspecification in Language Processing. Language and Linguistics Compass 3:111-127. https://doi.org/10.1111/j.1749-818x.2008.00104.x

Gergely G, Csibra G (2006) Sylvia's recipe: The role of imitation and pedagogy in the transmission of cultural knowledge. Roots of Human Sociality: Culture, Cognition, and Human Interaction 229-255.

Haun D, Rekers Y, Tomasello M (2014) Children Conform to the Behavior of Peers; Other Great Apes Stick With What They Know. Psychological Science 25:2160-2167. https://doi.org/10.1177/0956797614553235

Herrmann P, Legare C, Harris P, Whitehouse H (2013) Stick to the script: The effect of witnessing multiple actors on children's imitation. Cognition 129:536-543. https://doi.org/10.1016/i.cognition.2013.08.010

Hoehl S, Keupp S, Schleihauf $\mathrm{H}$ et al. (2019) 'Over-imitation': A review and appraisal of a decade of research. Developmental Review 51:90-108. https://doi.org/10.1016/j.dr.2018.12.002

Horner V, Whiten A (2005) Causal knowledge and imitation/emulation switching in chimpanzees (Pan troglodytes) and children (Homo sapiens). Animal Cognition 8:164181. https://doi.org/10.1007/s10071-004-0239-6

Keupp S, Behne T, Rakoczy H (2013) Why do children overimitate? Normativity is crucial. Journal of Experimental Child Psychology 116:392-406. https://doi.org/10.1016/i.jecp.2013.07.002

Kirby S, Cornish H, Smith K (2008) Cumulative cultural evolution in the laboratory: An experimental approach to the origins of structure in human language. Proceedings of the National Academy of Sciences 105:10681-10686. https://doi.org/10.1073/pnas.0707835105

Kumpfer KL, Alvarado R, Tait C, Turner C (2002) Effectiveness of school-based family and children's skills training for substance abuse prevention among 6-8-year-old rural children. Psychology of addictive behaviors 16:S65. https://doi.org/10.1037/0893164X.16.4S.S65Laland K (2017) Darwin's unfinished symphony. Princeton University Press, Princeton

Landis J, Koch G (1977) The Measurement of Observer Agreement for Categorical Data. Biometrics 33:159-174. https://doi.org/10.2307/2529310 
Legare C, Harris P (2016) The Ontogeny of Cultural Learning. Child Development 87:633-642. https://doi.org/10.1111/cdev.12542

Legare C, Nielsen M (2015) Imitation and Innovation: The Dual Engines of Cultural Learning. Trends in Cognitive Sciences 19:688-699. https://doi.org/10.1016/i.tics.2015.08.005

Legare C, Wen N, Herrmann P, Whitehouse H (2015) Imitative flexibility and the development of cultural learning. Cognition 142:351-361. https://doi.org/10.1016/j.cognition.2015.05.020

Lenth R, Lenth MR (2018) Package 'Ismeans.' The American Statistician 34:216-221

Lyons D, Young A, Keil F (2007) The hidden structure of overimitation. Proceedings of the National Academy of Sciences 104:19751-19756. https://doi.org/10.1073/pnas.0704452104

McGuigan N (2012) The role of transmission biases in the cultural diffusion of irrelevant actions. Journal of Comparative Psychology 126:150-160. https://doi.org/10.1037/a0025525

McGuigan N, Graham M (2009) Cultural transmission of irrelevant tool actions in diffusion chains of 3- and 5-year-old children. European Journal of Developmental Psychology 7:561-577. https://doi.org/10.1080/17405620902858125

McGuigan N, Whiten A (2009) Emulation and "overemulation" in the social learning of causally opaque versus causally transparent tool use by 23- and 30-month-olds. Journal of Experimental Child Psychology 104:367-381. https://doi.org/10.1016/j.jecp.2009.07.001

McGuigan N, Burdett E, Burgess $V$ et al. (2017) Innovation and social transmission in experimental micro-societies: exploring the scope of cumulative culture in young children. Philosophical Transactions of the Royal Society B: Biological Sciences 372:20160425. https://doi.org/10.1098/rstb.2016.0425

McGuigan N, Makinson J, Whiten A (2011) From over-imitation to super-copying: Adults imitate causally irrelevant aspects of tool use with higher fidelity than young children. British Journal of Psychology 102:1-18. https://doi.org/10.1348/000712610x493115

McGuigan N, Whiten A, Flynn E, Horner V (2007) Imitation of causally opaque versus causally transparent tool use by 3- and 5-year-old children. Cognitive Development 22:353364. https://doi.org/10.1016/i.cogdev.2007.01.001

Meltzoff A (2007) 'Like me': a foundation for social cognition. Developmental Science 10:126134. https://doi.org/10.1111/j.1467-7687.2007.00574.x

Meltzoff A (1988a) Infant imitation after a 1-week delay: Long-term memory for novel acts and multiple stimuli. Developmental Psychology 24:470-476. https://doi.org/10.1037/0012-1649.24.4.470 
762

763

764

765

766

767

768

769

770

771

772

773

774

775

776

777

778

779

780

781

782

783

784

785

786

787

788

789

790

791

792

793

Meltzoff A (1988b) Infant imitation after a 1-week delay: Long-term memory for novel acts and multiple stimuli. Developmental Psychology 24:470-476. https://doi.org/10.1037/0012-1649.24.4.470

Mesoudi A, Thornton A (2018) What is cumulative cultural evolution?. Proceedings of the Royal Society B: Biological Sciences 285:20180712. https://doi.org/10.1098/rspb.2018.0712

Moraru C, Gomez J, McGuigan N (2016) Developmental changes in the influence of conventional and instrumental cues on over-imitation in 3- to 6-year-old children. Journal of Experimental Child Psychology 145:34-47. https://doi.org/10.1016/i.jecp.2015.11.017

Morgan T, Uomini N, Rendell L et al. (2015) Experimental evidence for the co-evolution of hominin tool-making teaching and language. Nature Communications 6:1-8. https://doi.org/10.1038/ncomms7029

Nagell K, Olguin R, Tomasello M (1993) Processes of social learning in the tool use of chimpanzees (Pan troglodytes) and human children (Homo sapiens). Journal of Comparative Psychology 107:174-186. https://doi.org/10.1037/0735-7036.107.2.174

Neely RJ, Green JL, Sciberras E, et al (2016) Relationship between executive functioning and symptoms of attention-deficit/hyperactivity disorder and autism spectrum disorder in 6-8 year old children. Journal of autism and developmental disorders 46:3270-3280. https://doi.org/10.1007/s10803-016-2874-6

Nelder JA (1977) A reformulation of linear models. Journal of the Royal Statistical Society, Series A (General) 140: 48-77.

Nielsen M (2006) Copying actions and copying outcomes: Social learning through the second year. Developmental Psychology 42:555-565. https://doi.org/10.1037/0012$\underline{1649.42 .3 .555}$

Nielsen M (2018) The Social Glue of Cumulative Culture and Ritual Behavior. Child Development Perspectives 12:264-268. https://doi.org/10.1111/cdep.12297

Nielsen M, Tomaselli K (2010) Overimitation in Kalahari Bushman Children and the Origins of Human Cultural Cognition. Psychological Science 21:729-736. https://doi.org/10.1177/0956797610368808

Nielsen M, Cucchiaro J, Mohamedally J (2012) When the Transmission of Culture Is Child's Play. PLoS ONE 7:e34066. https://doi.org/10.1371/journal.pone.0034066

Nielsen M, Mushin I, Tomaselli K, Whiten A (2014) Where Culture Takes Hold: “Overimitation" and Its Flexible Deployment in Western, Aboriginal, and Bushmen Children. Child Development n/a-n/a. https://doi.org/10.1111/cdev.12265 
Ohnuma K, Aoki K, Akazawa A (1997) Transmission of Tool-making through Verbal and Nonverbal Communication: Preliminary Experiments in Levallois Flake Production. Anthropological Science 105:159-168. https://doi.org/10.1537/ase.105.159

Papanastasiou C (2002) TIMSS study in cyprus: Patterns of achievements in mathematics and science. Studies in Educational Evaluation 28:223-233. https://doi.org/10.1016/s0191-491x(02)80004-9

Patil SP, Patil PB, Kashetty M v (2014) Effectiveness of different tooth brushing techniques on the removal of dental plaque in 6-8 year old children of Gulbarga. Journal of International Society of Preventive \& Community Dentistry 4:113. https://doi.org/10.4103/2231-0762.138305

Putt S, Woods A, Franciscus R (2014) The role of verbal interaction during experimental bifacial stone tool manufacture. Lithic Technology 39:96-112. https://doi.org/10.1179/0197726114z.00000000036

Reader S, Bruce M, Rebers S (2007) Social learning of novel route preferences in adult humans. Biology Letters 4:37-40. https://doi.org/10.1098/rsbl.2007.0544

Subbotsky E (2004) Magical thinking in judgments of causation: Can anomalous phenomena affect ontological causal beliefs in children and adults?. British Journal of Developmental Psychology

22:123-152. https://doi.org/10.1348/026151004772901140

Tamariz M (2019) Replication and emergence in cultural transmission. Physics of Life Reviews 30:47-71. https://doi.org/10.1016/j.plrev.2019.04.004

Tamariz M, Kirby S, Carr J (2016) Cultural Evolution Across Domains: Language, Technology and Art. CogSci

Team RC, others (2012) R: A language and environment for statistical computing. 2012. Vienna, Austria: R Foundation for Statistical Computing 10

Tennie C, Call J, Tomasello M (2006) Push or Pull: Imitation vs. Emulation in Great Apes and Human Children. Ethology 112:1159-1169. https://doi.org/10.1111/j.1439$\underline{0310.2006 .01269 . x}$

Tennie C, Call J, Tomasello M (2009) Ratcheting up the ratchet: on the evolution of cumulative culture. Philosophical Transactions of the Royal Society B: Biological Sciences 364:2405-2415. https://doi.org/10.1098/rstb.2009.0052

Tennie C, Walter V, Gampe A et al. (2014) Limitations to the cultural ratchet effect in young children. Journal of Experimental Child Psychology 126:152-160. https://doi.org/10.1016/i.jecp.2014.04.006

Tomasello M (1994) The question of chimpanzee culture. In: Wrangham R, McGrew W, de Waal F, Heltne P (ed) Chimpanzee Cultures. Harvard University Press, Cambridge, MA, pp 301-317 
831

832

833

834

835

836

837

838

839

840

841

842

843

844

845

846

847

848

849

850

851

852

853

854

855

856

857

858

859

860

861

862

863

864

865

Tomasello M (1999) The cultural origins of human cognition. Harvard University Press, Cambridge, Mass.

Tomasello M (1999) The Human Adaptation for Culture. Annual Review of Anthropology 28:509-529. https://doi.org/10.1146/annurev.anthro.28.1.509

Tomassello M (2006) 10 Uniquely Human Cognition Is a Product of Human Culture. In: Levinson S, Jaisson P (ed) Evolution and Culture: A Fyssen Foundation Symposium. The MIT Press, Cambridge, Massachusetts, pp 203-217

Tomasello M (2016) The ontogeny of cultural learning. Current Opinion in Psychology 8:1-4. https://doi.org/10.1016/j.copsyc.2015.09.008

Tomasello M, Kruger A, Ratner H (1993) Cultural learning. Behavioral and Brain Sciences 16:495-511. https://doi.org/10.1017/s0140525x0003123x

Tomassello M (1990) Cultural transmission in the tool use and communicatory signaling of chimpanzees? In: Parker S, Gibson K (ed) "Language" and intelligence in monkeys and apes. Cambridge University Press, Cambridge [England]

Wen N, Herrmann P, Legare C (2016) Ritual increases children's affiliation with in-group members. Evolution and Human Behavior 37:54-60. https://doi.org/10.1016/j.evolhumbehav.2015.08.002

Whiten A, Ham R (1992) On the nature and evolution of imitation in the animal kingdom: reappraisal of a century of research. In: Slater PJB, Rosenblatt JS, Beer C, Milinski M (ed) Advances in the Study of Behavior. New York: Academic Press, pp 239-283

Whiten A, McGuigan N, Marshall-Pescini S, Hopper L (2009) Emulation, imitation, overimitation and the scope of culture for child and chimpanzee. Philosophical Transactions of the Royal Society B: Biological Sciences 364:2417-2428. https://doi.org/10.1098/rstb.2009.0069

Whiten A, Allan G, Devlin S et al. (2016) Social Learning in the Real-World: 'Over-Imitation' Occurs in Both Children and Adults Unaware of Participation in an Experiment and Independently of Social Interaction. PLOS ONE 11:e0159920. https://doi.org/10.1371/journal.pone.0159920

Winter B, Wieling M (2016) How to analyze linguistic change using mixed models, Growth Curve Analysis and Generalized Additive Modeling. Journal of Language Evolution 1:718. https://doi.org/10.1093/jole/lzv003

Wood L, Kendal R, Flynn E (2012) Context-dependent model-based biases in cultural transmission: children's imitation is affected by model age over model knowledge state. Evolution and Human Behavior 33:387-394. https://doi.org/10.1016/i.evolhumbehav.2011.11.010 
Woolley J (1997) Thinking about Fantasy: Are Children Fundamentally Different Thinkers and Believers from Adults?. Child Development 68:991-1011. https://doi.org/10.2307/1132282

Zwirner E, Thornton A (2015) Cognitive requirements of cumulative culture: teaching is useful but not essential. Scientific Reports 5:1-8. https://doi.org/10.1038/srep16781

871

872

873 Data availability

874 The datasets generated during and/or analysed during the current study are available in the 875 Zenodo repository, https://zenodo.org/record/4444854\#.YALWyOEYA2w

876

877 Competing interests

878 The authors declare no competing interests. 